\title{
Frequency stabilization at the kilohertz level of a continuous intracavity frequency- doubled singly resonant optical parametric oscillator
}

\author{
Oussama Mhibik, Thu-Hien My, David Pabouf, Fabien Bretenaker, ${ }^{*}$ and Cyril Drag \\ Laboratoire Aimé Cotton, CNRS-Université Paris Sud 11, 91405 Orsay Cedex, France \\ ${ }^{*}$ Corresponding author: Fabien.Bretenaker@lac.u-psud.fr
}

Received March 30, 2010; revised June 11, 2010; accepted June 12, 2010; posted June 25, 2010 (Doc. ID 126204); published July 7, 2010

\begin{abstract}
A continuous intracavity frequency-doubled singly resonant optical parametric oscillator (OPO) is stabilized to the side of the transmission peak of a medium finesse Fabry-Perot cavity. The narrow bandwidth of the frequency noise of this OPO allows this simple scheme to lead to a stability of a few kilohertz with respect to the locking etalon. The system, operating in the visible domain, remains locked for more than $1 \mathrm{~h}$. (c) 2010 Optical Society of America OCIS codes: $190.4970,190.2620$
\end{abstract}

Rare-earth ions embedded in crystalline matrices are promising candidates for the realization of optically controlled quantum computers [1-3] and quantum memories $[\underline{4}, 5]$. They are even considered to achieve the strong coupling regime of cavity quantum electrodynamics in monolithic optical resonators [6]. All these applications use the remarkably long coherence lifetimes of these ions. For example, homogeneous linewidths smaller than $1 \mathrm{kHz}$ have been observed for the optical transitions of europium and praseodymium $[\underline{7,8]}$. Unfortunately, the optical transitions of these two latter ions lie at 579 and $606 \mathrm{~nm}$, respectively, in the domain where diode lasers are not readily available. Moreover, some coherent processes used to drive these systems [9] require a laser whose short-term linewidth is smaller than the homogeneous linewidth of the considered ion, i.e., in the kilohertz domain. Up to now, this has been possible only using dye lasers [10]. However, due to the high-frequency noise of the dye jet, these systems can be stabilized to the kilohertz level only with an intracavity phase modulator [11]. Recent works on quantum memories and quantum information processing have stressed the importance of laser frequency stability $[3, \underline{3}, \underline{12}]$. We have recently shown that an intracavity frequency doubled singly resonant optical parametric oscillator (SHG-SROPO, where SHG stands for second-harmonic generation) is a good candidate to replace $\mathrm{cw}$ dye lasers [13]. In particular, the bandwidth of the frequency fluctuations of such a source is much narrower than that for a dye laser [13]. This is consistent with several reports showing that $\overline{\mathrm{SROPO}}$ can be relatively easily stabilized at the megahertz level $[14,15]$ and below $[16,17]$. Our aim here is to take advantage of the small bandwidth of the frequency fluctuations of this system to prove that a simple stabilization of the frequency on the side of the transmission fringe of a Fabry-Perot (FP) cavity permits to reach the kilohertz frequency stability range. Although subkilohertz frequency stabilization of doubly resonant OPOs has already been achieved [18], this has, to our best knowledge, never been achieved in the case of SROPOs.

Our experimental setup is schematized in Fig. 1. The OPO is pumped at $532 \mathrm{~nm}$ by a $10 \mathrm{~W}$ single-frequency Verdi laser. It is based on a 30-mm-long MgO-doped periodically poled stoichiometric lithium tantalate (PPSLT) crystal $\left(d_{\text {eff }} \simeq 11 \mathrm{pm} / \mathrm{V}\right)$ manufactured and coated by HC Photonics. This single-grating crystal $(7.97 \mu \mathrm{m}$ period $)$ is antireflection coated for the pump, signal, and idler. It leads to quasi-phase-matching for an idler wavelength in the $1200-1400 \mathrm{~nm}$ range. The OPO ring cavity is $1.15 \mathrm{~m}$ long and consists of four mirrors. The two mirrors sandwiching the PPSLT crystal have a $150 \mathrm{~mm}$ radius of curvature. The two other mirrors are plane. This cavity is resonant for the idler only. The estimated waist of the idler beam at the middle of the PPSLT crystal is $37 \mu \mathrm{m}$. The pump beam is focused to a $53 \mu \mathrm{m}$ waist inside this crystal. All mirrors are designed to exhibit a reflectivity larger than $99.8 \%$ between 1.2 and $1.4 \mu \mathrm{m}$ and a transmission larger than $95 \%$ at $532 \mathrm{~nm}$ and between 850 and $950 \mathrm{~nm}$.

A 25-mm-long beta-barium borate (BBO) crystal is inserted between the two plane mirrors, i.e., at the second waist of the cavity. It is antireflection coated for the idler and the second-harmonic wavelengths. A 1.5-mm-thick etalon with a finesse $F=3$ is also inserted in this leg of the cavity. In these conditions and with the PPSLT crystal heated at $T=103{ }^{\circ} \mathrm{C}$, the system oscillates at an idler wavelength of $1204 \mathrm{~nm}$ with a pump threshold equal to $800 \mathrm{~mW}$. At a pump power equal to $3.4 \mathrm{~W}$, the SHG-SROPO emits a single-frequency orange beam at $602 \mathrm{~nm}$ with a $90 \mathrm{~mW}$ output power (only a few milliwatts

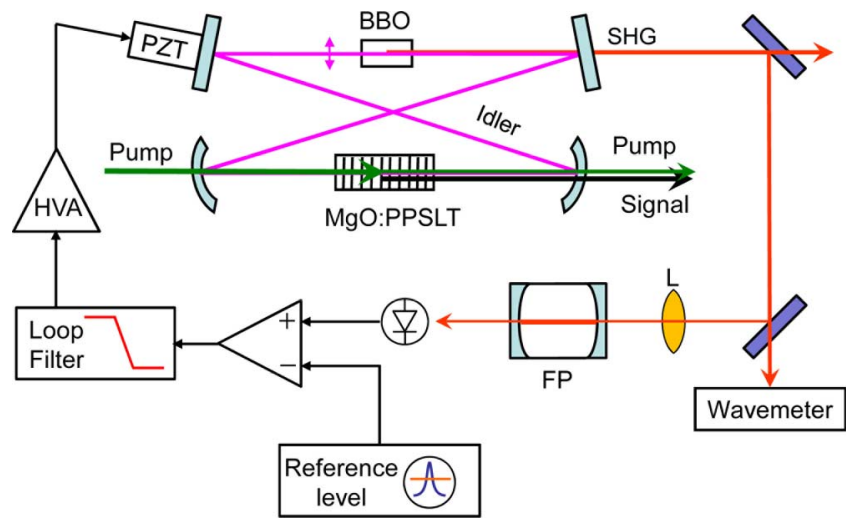

Fig. 1. (Color online) Experimental setup. HVA, high-voltage amplifier; PZT, piezoelectric transducer; L, focusing lens; FP, Fabry-Perot cavity. 
at $579 \mathrm{~nm}$, the mirrors being not optimized for this wavelength).

To measure the frequency fluctuations of this source, we send a part of the orange beam to a $750 \mathrm{MHz}$ free spectral range (FSR) confocal FP cavity with a rather low finesse $F=60$. We manually maintain the OPO frequency on the side of the transmission peak of the cavity and record the transmitted signal for $1 \mathrm{~s}$ with a sampling frequency of $2 \times 10^{5} \mathrm{~Hz}$. The Fourier transform of this signal leads to the frequency-noise spectrum of Fig. 2 [19]. The frequency standard deviation obtained from the square root of the area below the spectrum of Fig. 2 is equal to $400 \mathrm{kHz}$. This spectrum also shows that the frequency noise mainly occurs for frequencies below $1 \mathrm{kHz}$. This allows us to expect a simple frequency locking technique to strongly reduce this noise. For comparison, we measure the frequency noise of the pump laser using the same FP cavity that has a finesse $F=16$ at $532 \mathrm{~nm}$ (see Fig. 2), leading to a standard deviation of $1.2 \mathrm{MHz}$.

To stabilize the OPO, we use the servo loop of Fig. 1. As a frequency reference, we use another FP cavity of FSR equal to $1 \mathrm{GHz}$ with a finesse $F=100$. The transmission of this FP cavity is subtracted from a reference and filtered by an adjustable proportional-integrator (PI) filter (Precision Photonics model LB1005). It is then amplified by the high-voltage amplifier (HVA) (Piezomechanik model SVR150-3) and applied to a piezoelectric transducer (Piezomechanik model PSt 150/10 × 10/2, resonance frequencies above $20 \mathrm{kHz}$ ) carrying a cavity mirror weighing $6 \mathrm{~g}$. We experimentally checked using an interferometer that the combination of this HVA and this transducer with the mirror glued on it behaves like a first-order low-pass filter with a $300 \mathrm{~Hz} 3 \mathrm{~dB}$ bandwidth. Moreover, the phase introduced by these elements changes by only $90^{\circ}$ between DC and $3 \mathrm{kHz}$. With the parameters of the loop filter chosen here (PI corner frequency equal to $10 \mathrm{kHz}$, no lowfrequency gain limit, proportional gain equal to $0 \mathrm{~dB}$ ), the open-loop transfer function of the servo loop exhibits a roll-off equal to $20 \mathrm{~dB} /$ decade between DC and $250 \mathrm{~Hz}$, $35 \mathrm{~dB} /$ decade between $250 \mathrm{~Hz}$ and $10 \mathrm{kHz}$, and again $20 \mathrm{~dB} /$ decade above $10 \mathrm{kHz}$. The $0 \mathrm{~dB}$ gain frequency is about $10 \mathrm{kHz}$.

In these conditions, the SHG-SROPO can be locked to the cavity and the error signal, processed as in Fig. 2, leads to the noise spectrum of Fig. 3. By comparison with Fig. 2 , the noise is strongly reduced and is almost white except

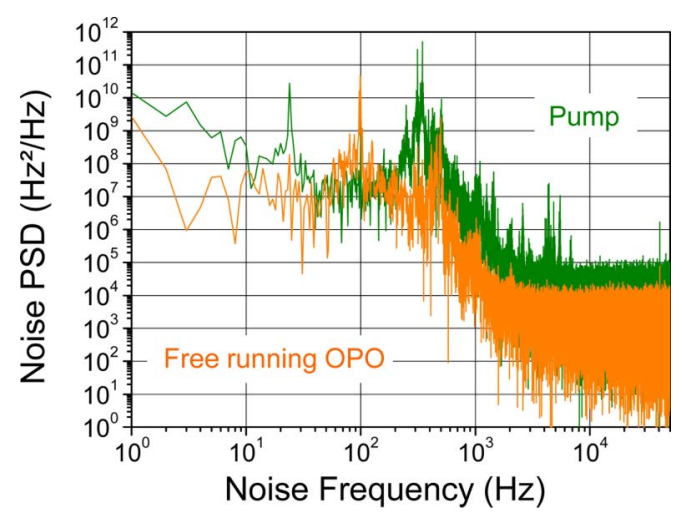

Fig. 2. (Color online) Spectrum of the frequency fluctuations of the free-running SHG-SROPO and of the pump laser.

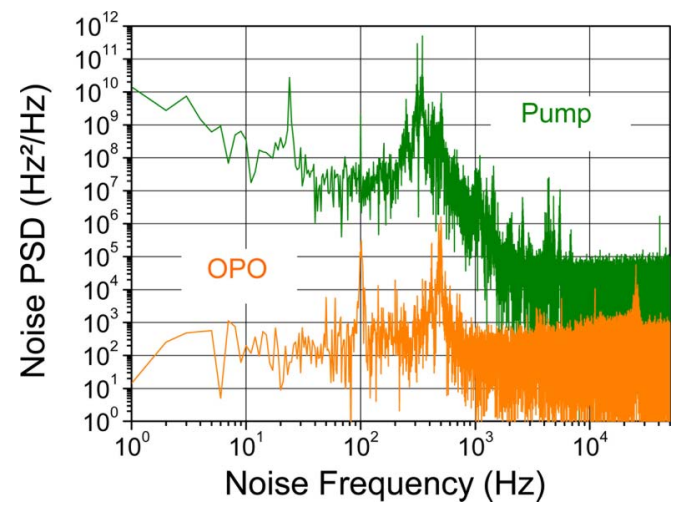

Fig. 3. (Color online) Spectrum of the relative frequency fluctuations of the servo-locked SHG-SROPO and of the pump laser.

for a few resonances between 100 and $1 \mathrm{kHz}$. These peaks are due either to electrical perturbations (at $100 \mathrm{~Hz}$ ) or to what remains of the fluctuations of the pump frequency (between 300 and $500 \mathrm{~Hz}$ ). However, by comparison with the spectrum of the Verdi laser (see Fig. 3), one sees that most of the frequency noise of the pump has been eliminated from the SHG-SROPO. This proves that our choice of a singly resonant architecture indeed permits us to stabilize the resonant beam (here the idler) below the pump laser frequency noise level and, consequently, to dump this noise into the nonresonating beam (here the signal). From Fig. 3, we extract an rms deviation of the frequency of the SHG-SROPO of $4 \mathrm{kHz}$ with respect to the cavity resonance. This means that the relative frequency stability of the idler is equal to $2 \mathrm{kHz}$. This is to be compared with the value of $1.2 \mathrm{MHz}$ obtained for the pump laser. The main part of the remaining frequency noise of our SHG-SROPO is due to the noise floor between $10^{2}$ and $10^{3} \mathrm{~Hz}^{2} / \mathrm{Hz}$. This floor is mainly due to the detector and the HVA. We also checked experimentally that the contributions of the intensity noises of the pump and the OPO to the spectra of Figs. 2 and 3 are negligible. To further reduce the noise floor and, consequently, the linewidth of the SHG-SROPO, we need to reduce the amplifier noise but also to use a higher-finesse cavity. However, this would make the simple scheme used here unstable and would consequently oblige us to implement a PoundDrever-Hall-type servo loop [20].

Stabilizing the frequency of the SHG-SROPO also stabilizes its intensity, as evidenced in Fig. 4. The rms

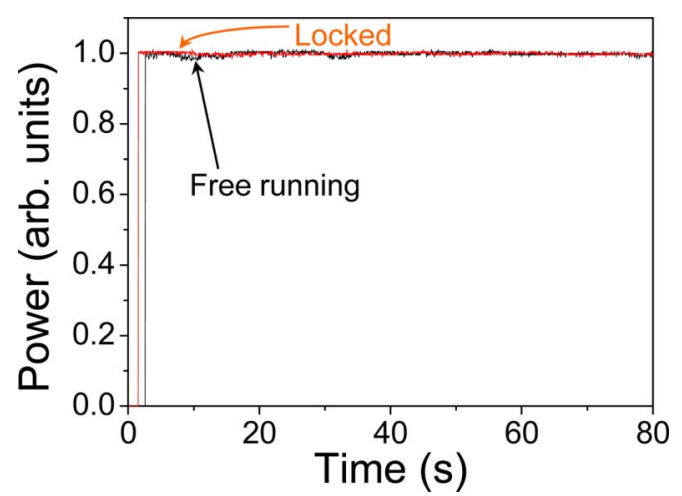

Fig. 4. (Color online) Time evolution of the output power of the locked and unlocked SHG-SROPO. 


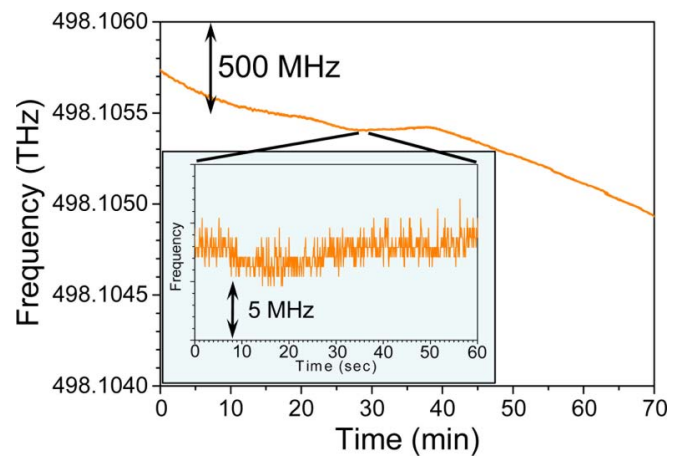

Fig. 5. (Color online) Time evolution of the frequency of the SHG-SROPO over more than $1 \mathrm{~h}$. The OPO remains locked to the cavity while it thermally drifts. The inset is a zoom lasting 1 min.

fluctuations of the power during $1 \mathrm{~min}$ are equal to $0.5 \%$ (respectively, 0.3\%) when the SHG-SROPO is unlocked (respectively, locked). Moreover, the SHG-SROPO remains locked during relatively long periods of time. Figure 5 shows an example lasting more than $1 \mathrm{~h}$. The frequency of the orange beam is measured using a $0.02 \mathrm{pm}$ resolution lambdameter. We can see that the SHG-SROPO frequency follows the thermal drift of the FP cavity (the data of Fig. 5 are compatible with a change of temperature of about $1{ }^{\circ} \overline{\mathrm{C}}$ in $1 \mathrm{~h}$ ). In particular, the inset shows that the short-term variations of the frequency are below the resolution of the lambdameter. Of course, long-term stabilization of the SHG-SROPO is feasible using either a thermally stabilized cavity and/or an atomic reference [21].

In conclusion, we have shown that the intrinsically low-frequency nature of the frequency noise of a SROPO makes it very easy to stabilize to the kilohertz level, i.e., well below the frequency noise of the pump laser. In the present case, this has been applied to an SHG-SROPO, thus providing a red/orange source for quantum information processing applications using europium and praseodymium ions at cryogenic temperatures. In the future, we plan to use a Pound-Drever-Hall stabilization scheme with a higher-finesse cavity to decrease the linewidth down to the hertz level [22] .

This work is supported by the Triangle de la Physique.

\section{References}

1. J. J. Longdell, M. J. Sellars, and N. B. Manson, Phys. Rev. Lett. 93, 130503 (2004).

2. J. J. Longdell and M. J. Sellars, Phys. Rev. A 69, 032307 (2004).

3. L. Rippe, M. Nilsson, S. Kröll, R. Klieber, and D. Suter, Phys. Rev. A 71, 062328 (2005).

4. B. Kraus, W. Tittel, N. Gisin, M. Nilsson, S. Kröll, and J. I. Cirac, Phys. Rev. A 73, 020302(R) (2006).

5. M. Afzelius, I. Usmani, A. Amari, B. Lauritzen, A. Walther, C. Simon, N. Sangouard, J. Minář, H. de Riedmatten, N. Gisin, and S. Kröll, Phys. Rev. Lett. 104, 040503 (2010).

6. D. L. McAuslan, J. J. Longdell, and M. J. Sellars, Phys. Rev. A 80, 062307 (2009).

7. R. W. Equall, Y. Sun, R. L. Cone, and R. M. Macfarlane, Phys. Rev. Lett. 72, 2179 (1994).

8. R. W. Equall, R. L. Cone, and R. M. Macfarlane, Phys. Rev. B 52, 3963 (1995).

9. F. de Seze, F. Dahes, V. Crozatier, I. Lorgeré, F. Bretenaker, and J.-L. Le Gouët, Eur. Phys. J. D 33, 343 (2005).

10. B. Julsgaard, A. Walther, S. Kröll, and L. Rippe, Opt. Express 15, 11444 (2007).

11. J. Helmcke, S. A. Lee, and J. L. Hall, Appl. Opt. 21, 1686 (1982).

12. I. Roos and K. Mølmer, Phys. Rev. A 69, 022321 (2004).

13. T. H. My, C. Drag, and F. Bretenaker, Opt. Lett. 33, 1455 (2008).

14. J.-M. Melkonian, T. H. My, F. Bretenaker, and C. Drag, Opt. Lett. 32, 518 (2007).

15. S. Zaske, D.-H. Lee, and C. Becher, Appl. Phys. B 98, 729 (2010).

16. U. Strössner, J.-P. Meyn, R. Wallenstein, P. Urenski, A. Arie, G. Rosenman, J. Mlynek, S. Schiller, and A. Peters, J. Opt. Soc. Am. B 19, 1419 (2002).

17. E. V. Kovalchuk, T. Schuldt, and A. Peters, Opt. Lett. 30, 3141 (2005).

18. R. Al-Tahtamouni, K. Bencheikh, R. Storz, K. Schneider, M. Lang, J. Mlynek, and S. Schiller, Appl. Phys. B 66, 733 (1998).

19. W. Vassen, C. Zimmermann, R. Kallenbach, and T. W. Hänsch, Opt. Commun. 75, 435 (1990).

20. R. W. P. Drever, J. L. Hall, F. V. Kowalski, J. Hough, G. M. Ford, A. J. Munley, and H. Ward, Appl. Phys. B 31, 97 (1983).

21. J. Helmcke, J. J. Snyder, A. Morinaga, F. Mensing, and M. Gläser, Appl. Phys. B 43, 85 (1987).

22. Ch. Salomon, D. Hils, and J. L. Hall, J. Opt. Soc. Am. B 5, 1576 (1988). 